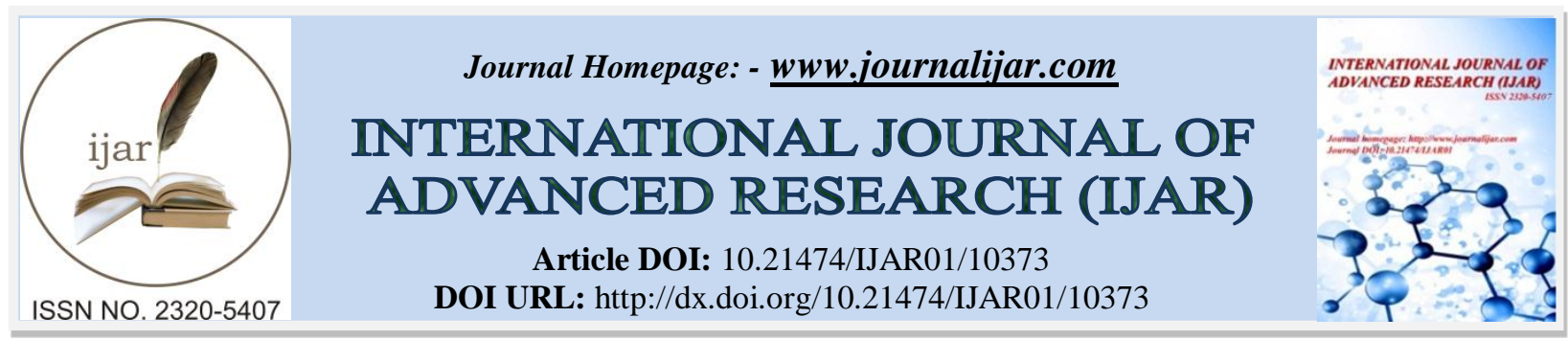

RESEARCH ARTICLE

\title{
MANAGEMENT OF AKV BY TELEMEDICINE : STUDY ABOUT 200 PATIENTS
}

\author{
Jaouad Nguadi ${ }^{1}$, Hajar Elouartassi ${ }^{1}$, Badre Elboussaadani ${ }^{1}$, Bouthayna Mesmoudi ${ }^{1}$, Anas Hadari ${ }^{3}$, \\ Ratiba Jennane $^{1}$, H.Zineddine ${ }^{3}$, Mounir Ababou ${ }^{2}$, Meryem Bennani ${ }^{1}$, Jamal Kheyi ${ }^{1}$, Najat Mouine ${ }^{1}$, \\ Abdelmajid Bouzerda ${ }^{1}$, Hicham Bouzelmate ${ }^{1}$, Ilyass Asfalou ${ }^{1}$, Maha Raissouni ${ }^{1}$, Nabil Berrada ${ }^{1}$, Zouhair \\ Lakhal $^{1}$, Aatif Benyass ${ }^{1}$ and Ali Chaib ${ }^{1}$ \\ 1. Heart Center- AVICENNA UHC - Rabat. \\ 2. Hematology- AVICENNA UHC - Rabat. \\ 3. Cardiology-AVICENNA Hospital- Marrakech.
}

\section{Manuscript Info}

Manuscript History

Received: 30 November 2019

Final Accepted: 31 December 2019

Published: January 2020

\section{Abstract}

\section{Introduction:-}

Telemedicine is defined by the practice of medicine at a distance using means of information and communications technology. Given the importance of clinical examination, cardiology is not very well suitedto telemedicine. However, just like inall medical specialties, telemedicine facilitates and supplements clinical medicine.

The Diagnosis Center of Cardiology in Rabat welcomes patients from different regions of Morocco. The longdistance travel, difficulty of access to care in rural Morocco, transportation problems, and delayed appointments all make follow-up of patients a very difficult task, therefore, many patients miss their consultation appointments. To avoid this problem, we followed our patients by social messaging platforms, thenjudged the effectiveness of this methoda year later.

\section{Materials and Methods:-}

This is a prospective, comparative analytic study of 200 patients underoral anticoagulant therapy based on vitamin $\mathrm{K}$ antagonists (VKA), followed in cardiology consultation, all of whom come from rural areas where access to care is difficult.

These 200 patients were followed during 1 year in consultation, 100 of them (group 1)in standard consultation according to the appointments elaborated by the computerized system of the Diagnosis Center. The other 100 (group 2) were followed, moreover, by their doctor via telephone.

Indications for oral anticoagulation in the 1st group were the following: $51 \%$ for atrial fibrillation, $18 \%$ for mitral prosthesis, 23\% for aortic prosthesis, $8 \%$ for another indication (other heart rhythm disorders, pulmonary embolism, other thromboembolic disorders ...). For the second group, 54\% had atrial fibrillation, 19\% mitral prosthesis, 20\% aortic prosthesis, and 7\% had other thromboembolic disorders. 


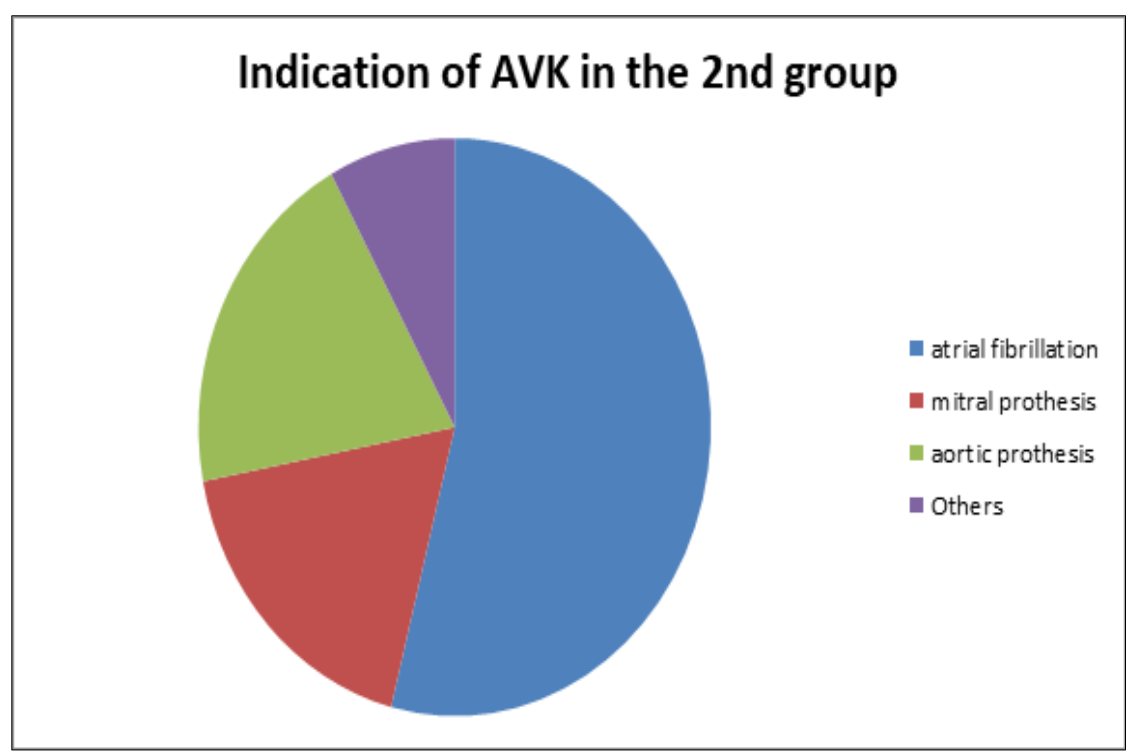

Figure 1:- Indications for oral anticoagulation in both groups.

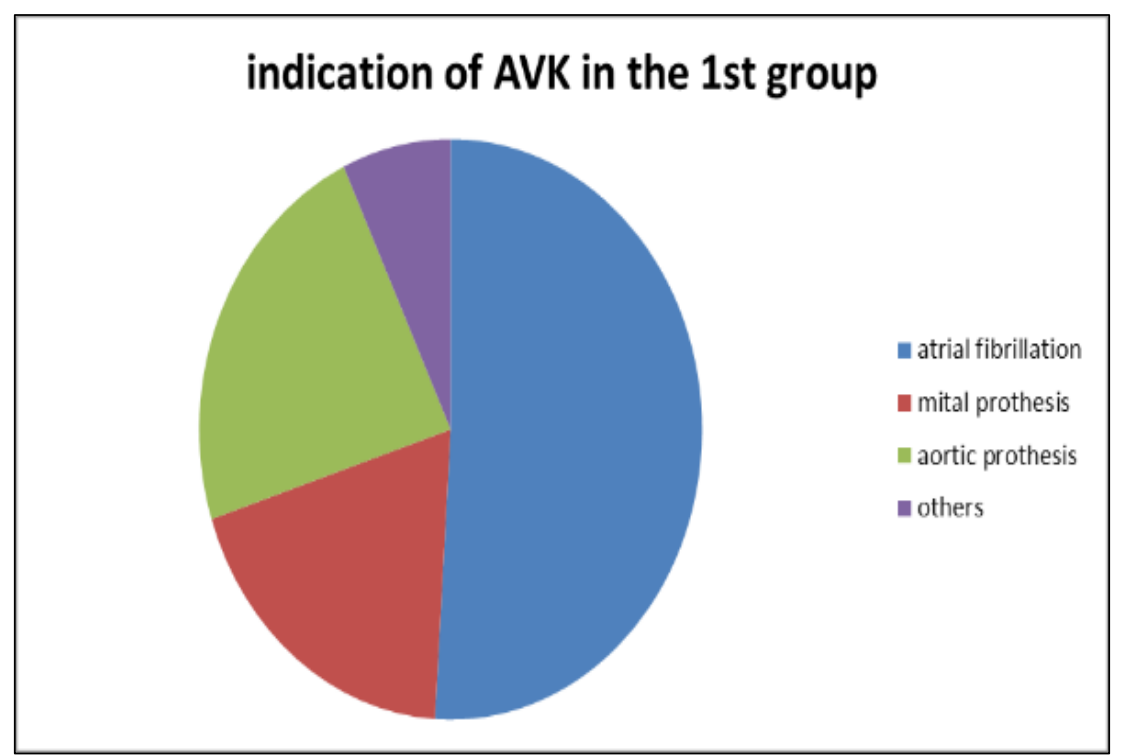

Figure 2:- INR assessments in both groups.

One year later, the evaluation of the management quality was based on:

1. One-year INR assessments:our study was carried out in collaboration with the hematology laboratory, all our patients carried out their biological testsat the hospital laboratory under the same conditions.

2. Presented complications: the evaluation of complications was based on the number, severity and rapidity of management of com plications particularly hemorrhagic and embolic accidents.

3. Patients' personal expenses: transportation expenses were judged on the number of trips made by each patient, and expenses of clinical managementparticularly hospital stay costs and management of complications.

\section{Results:-}

\begin{tabular}{|l|l|l|}
\hline & group 1 & group 2 \\
\hline Number of INR tests & 500 & 1305 \\
\hline INR per patient per month & 0.41 & 1.09 \\
\hline
\end{tabular}




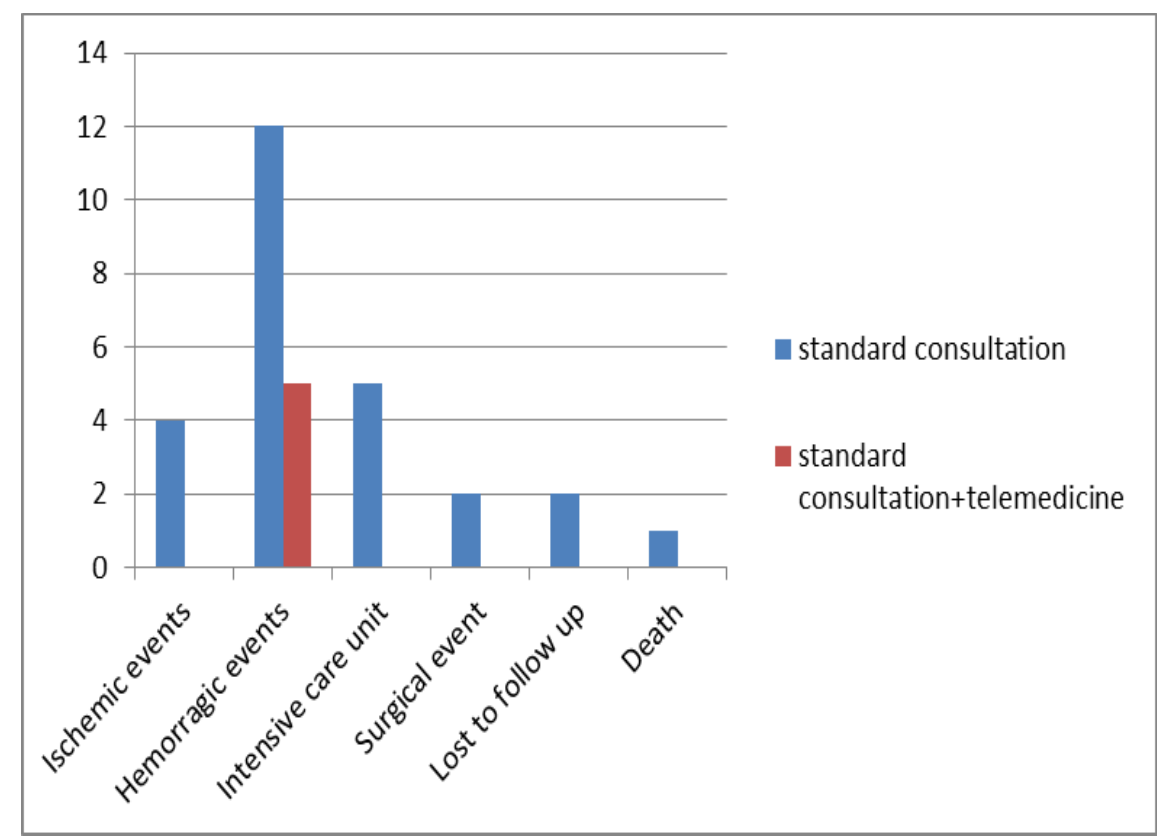

Figure 3:- Occurring complications in both groups.

\begin{tabular}{|l|l|l|l|}
\hline & & Group 1 & Group 2 \\
\hline \multirow{3}{*}{ Hospitalizations } & Number & 6 & 0 \\
\cline { 2 - 4 } & & & \\
\cline { 2 - 4 } Surgery & Expenses $(€)$ & 13000 & 0 \\
\hline \multirow{3}{*}{ Transport } & Number & 2 & 0 \\
\cline { 2 - 4 } & Expenses $(€)$ & 18000 & 0 \\
\hline Total amount of expensesin $€$ & Number/patient/year & 11 & 7 \\
\cline { 2 - 4 } & Expenses $(€)$ & $220 € / \mathrm{pt} / \mathrm{y}$ & $140 € / \mathrm{pt} / \mathrm{y}$ \\
\hline
\end{tabular}

Figure 4:- Expenses in both groups.

Patients of the 2nd group (group followed by telephone) were well anticoagulated most of the times with more frequent INR tests; 1305 INR performed by the 2 nd group (1.09 per patient per month) compared with 500 INR by the 1st group ( 0.41 per patient per month). The number of complications was lower in the 2 nd group as 5 patients presented a symptomatic overdose to anticoagulants and have evolved well, versus 12 for the 1st group, 4 of whom required hospitalization in the Intensive Care Unit for serious bleeding events, one of them has deceasedand another had a hemostasis gastrectomy.Four patients in the first group had embolic events: 1 ischemic stroke with sequelae, 1 transient ischemic attack (TIA), and 2 prosthetic thromboses with one treated medically and the other surgically. Transportation expenses of the 2nd group were much smaller.

\section{Discussion:-}

Even by simple means, telemedicine has shown its potential contribution in terms of mortality, morbidity, and in number of avoided hospitalizations. Its impact in terms of health economics is documented. [1]

A close monitoring of patients improves the prognosis of chronic diseases and ensures the proper use of chronic treatments. Based on monitoring by telephone messaging and social networks, our study showed a clear reduction in hemorrhagic and embolic complications and a good efficacy and safety in the use of oral anticoagulants in the group followed by telemedicine, with also better economic savings in therapeutic expenditure.[2]

Through this experience of our study, we may be witnessing the birth of the "medicine of tomorrow". Indeed, in the field of chronic diseases, in terms of their epidemiology and the foreseeable deficit of time and resources, we need to better monitor and educate. 
Telemedicine based on social networks and messaging platforms is more comfortable for the treating physician than telephone calls.

In their review on follow-up by telephone or telemonitoring of HF patients, Inglis et al. show that telemedicine has an effect on all-cause mortality, with a significant reduction of 34\% ( $\mathrm{p}<0.0001$ ). In this study, it is also put forward a reduction in re-hospitalizations for $\mathrm{HF}$ of $20 \%$, an improvement in the quality of life of patients, costs of care, and a good acceptability of the system. [3.4]

In the meta-analysis of Anker et al. studies were analyzed as part of a comparison between the effects of telemonitoring compared to usual care (non-invasive telemedicine). [4,5] In this work, telemonitoring showed the reduction of the following elements: all-cause mortality ( $10.4 \%$ versus $15.4 \% ; \mathrm{p}<0.0001$ ), admission to hospital for all causes $(47.2 \%$ versus $52.1 \% ; \mathrm{p}=0.02)$, hospitalization linked to chronic HF $(22.4 \%$ versus $28.5 \%$; $\mathrm{p}=$ 0.008).[5,6,7]

\section{Conclusion:-}

Telemedicine based on social networks and messaging platforms is more comfortable for the treating physician than telephone calls.

Health policy should consider telemedicine as a solution for a better managementof the Moroccan population and as a solution for health problems in the framework of the citizen approximation initiative.

\section{Bibliography:-}

1. Rosen D, McCall JD, Primack BA. Telehealth protocol to prevent readmission among high-risk patients with congestive heart failure. Am J Med 2017;130:1326-30. Burdese E, Testa M, Raucci P, et al. Usefulnessof a telemedicine program in refractory older congestive heart failure patients. Diseases 2018

2. Feltner $\mathrm{C}$, Jones $\mathrm{CD}$, Cené $\mathrm{CW}$, et al.Transitional care interventions to prevent readmissions for persons with heart failure: asystematic review and meta-analysis.

3. Martínez-González NA, Berchtold P, Ullman K, et al. Integrated care programmes for adults with chronic conditions: a meta-review. Int J Qual Health Care.

4. Achelrod D. Policy expectations and reality of telemedicine - a critical analysis of health care outcomes, costs and acceptance for congestive heart failure. J TelemedTelecare

5. Pandor A, Thokala P, Gomersall T, et al. Home telemonitoring or structured telephone support programmes after recent discharge in patients with heart failure: systematic review and economic evaluation. Health Technol Assess.

6. Fédération italienne des cliniques d'anticoagulant. Guide sur le traitement anticoagulant par voie orale. 1re partie. STV 1998; 10: 291-313

7. Fédération italienne des cliniques d'anticoagulant. Guide sur le traitement anticoagulant. 